Please do not remove this page

RMIT

UNIVERSITY

\title{
Explicit local segmentation based impulsive noise reduction for color images
}

Phu, Meing; Tischer, Peter; Wu, Henry

https://researchrepository.rmit.edu.au/esploro/outputs/9921859365801341/filesAndLinks?institution=61RMIT_INST\&index=null

Phu, M., Tischer, P., \& Wu, H. (2005). Explicit local segmentation based impulsive noise reduction for color images. Proceedings of 2005 International Symposium on Intelligent Signal Processing and Communication Systems, 657-660. https://doi.org/10.1109/ISPACS.2005.1595495

Published Version: https://doi.org/10.1109/ISPACS.2005.1595495

Repository homepage: https://researchrepository.rmit.edu.au

(c) 2005 IEEE. Personal use of this material is permitted. However, permission to reprint/republish this material for advertising or promotional purposes or for creating new collective works for resale or redistribution to servers or lists, or to reuse any copyrighted component of this work in other works must be obtained from the IEEE.

Downloaded On 2023/04/26 22:28:14 +1000 


\title{
EXPLICIT LOCAL SEGMENTATION BASED IMPULSIVE NOISE REDUCTION FOR COLOR IMAGES
}

\author{
Mieng Q. Phu ${ }^{1}$, Peter E. Tischer ${ }^{1}$ and Hon R. Wu ${ }^{2}$ \\ ${ }^{I}$ Clayton School of Information Technology, Monash University, Clayton Campus, VIC, Australia. \\ Mieng.Quoc.Phu@infotech.monash.edu.au \\ Peter.Tischer@csse.monash.edu.au \\ ${ }^{2}$ Software and Network Engineering, RMIT University, City Campus, VIC, Australia. \\ henry.wu@rmit.edu.au
}

\begin{abstract}
A family of local segmentation vector filters for color image noise suppression and detail preservation is proposed. Most state-of-the-art filters alleviate impulse noise well but tend to destroy thin lines, edges and fine image details. The proposed filters facilitate local segmentation to preserve image structures and noise suppression. First the K-VMF is developed and used for local segmentation, and then a selection of vector filters is used to reconstruct the current pixel. In addition, once pixels have been marked as being noisy, their values are not used in processing subsequent pixels. The proposed filters also demonstrated acceptable results for both objective and subjective assessments.
\end{abstract}

\section{INTRODUCTION}

In the specific area of color image restoration, filters are either categorized as component-wise or multivariate [1]. Component-wise methods deal with each channel separately, whereas multivariate filters process color pixels as vectors. Since color channels are strongly correlated, vector filters tend to perform better and produce fewer artifacts such as color bleeding and distortions. When dealing with impulsive noise, where the original pixels are completely replaced by random noise, some people advocate that the most efficient filtering approach is based on the vector order-statistic theory [2].

One of the most popular vector filters is the Vector Median filter (VMF) [3]. Other filters include Vector Direction filter (VDF/GVDF) [4], Directional Distance filter (DDF) [5], Hybrid Directional filter (HDF/AHDF) [6] and Adaptive Nearest Neighbour filter (ANNF) [7]. These classic filters remove impulse noise adequately but they tend to introduce new artifacts to image structures such as blurring, smearing and shifting. This happens because they do not classify pixels as been clean, noise, blotch of noises, or high image detail. The Multiple Window Configuration (MWC) [8] solves this drawback by the use of detection and switching. However, this filter depends on the reference image and fine tuning on specific images to achieve optimal results for that image. Another switch based filter is Neighbour Adaptive Vector filter (NAVF) [9]. All of these filters implicitly or explicitly assumed that the current window is homogeneous. This is true on most parts of the image, but there are edges, thin lines and outliers as well. Thus, the assumption of homogeneity will lead to the removal of not only noise but also the image structure. The Peer Group filter (PGF) [10] uses Fisher's Discriminant to segment pixels explicitly into groups with similar intensity and to reconstruct using the VMF and a weighting function.

\section{VECTOR MEDIAN FILTER}

VMF is one of the most efficient and popular filter because of its simplicity and low computational cost. It is extensively used, for example, as an impulse detector, and in hybrid and switch based filters.

Let $\underset{\sim}{x}$ be as a multichannel sample vector and $\mathrm{W}$ be the window $\left(x_{1}, x_{2}, \ldots, x_{n}\right)$. For pixel $x_{i}$, its total distance to the other pixels in the window is given (1)

$$
d_{w}\left(x_{i}\right)=\sum_{j=1}^{n} d\left(x_{i}, x_{j}\right)
$$

The distance $d\left(x_{i}, x_{j}\right)$ is often the Euclidean distance. Let the $d_{w}\left(x_{i}\right)$ be sorted in ascending order to produced $\left\{d_{1}, d_{2}, \ldots, d_{n}\right\}$. The pixel associated with $d_{1}$ is the most 'inlying' value and is chosen as the vector median (VM) and used as the output of the VMF.

Although the VMF can remove most impulsive noise, it tends to destroy image structure and blur the image as in Figure 1. When there is no noise within an image the VMF will destroy thin lines and edges. The VMF and its 
variants may destroy underlying image structure when the mask contains pixels from more than 1 segment. For example, suppose the mask contains 9 pixels which belong to two segments. The VM will be chosen from the pixels which belong to the segment which has more members in the mask. Thus, structure like corners and thin lines, which only have a small number of pixels in the mask, get erased. For a $3 \times 3$ mask, if the centre pixel is part of a segment whose intersection with the mask contains at most 4 pixels, the centre pixel will be replaced by a pixel from the other segment. Any feature whose intersection with the $3 \times 3$ mask never has more than 4 pixels will be completely erased. These drawbacks not only occur with the VMF but also with other vector filters as well. The motivation of this paper is to overcome these drawbacks by using local segmentation.

\section{FORMULATION OF LOCAL SEGMENTATIION VECTOR FILTERS (LSVF)}

The structure of the proposed filter is in Figure 2. It comprised of the homogeneity detection, the local segmentation process and the vector filters for reconstruction.

\subsection{Homogeneity Detection}

If equation (2) is true, the region is classified as being homogeneous and no filter is required. Otherwise, it will be treated as containing two segments. The threshold $\delta_{R}=55$ is chosen from [9].

$$
d_{n}-d_{1} \leq \delta_{R}
$$

\subsection{Local Segmentation (LS)}

In color image processing, the K-median method can extended to K-VMF for local segmentation in the RGB color space.

Since we are only trying to find two classes, $k=2$. The algorithm is as follows:

1) Choose two initial vectors to represent each class.

2) Assign each pixel in the mask to the representative vector which is closer.

3) Re-compute the representative vector for a class as the VM of the pixels in that class.

4) Repeat steps 2 to 4.

For initial vectors, there are two ways. One is to choose the two pixels in the mask which are the furthest apart (TYPE1). Another is to choose the centre pixel (CP) as one initial value and the pixel at the furthest distance from it is another (TYPE2). This latter method will require less computation than the first. In additional, if the $\mathrm{CP}$ is the sole pixel in the segment then it is considered to be noise.

\subsubsection{Pixel Marking (PM)}

When images are processed by rows, by the time a pixel is to be processed pixels in rows before the current row and in column of the current row up to but not including the current pixel, are known. It can be remembered whether these pixels were 'noisy' or 'clean'. For a $3 \times 3$ mask, 4 of the 9 pixels have already been processed and they will only be used in the LS process if they were 'clean'. This is shown in Figure 3.

\subsection{Vector Filtering (VF)}

If we have split the pixels into two segments our program allows any vector filter (E.g. VMF, VDF, DDF, ANNF, etc) to be applied to the segment containing the current pixel, creating a whole family of Local Segment Vector Filter (LSVF). The vector filter will only be applied if the segment is considered to be 'valid'. If the segment has 3 or more pixels, it is considered as 'valid'. If it has 2 pixels or fewer, it is considered 'invalid' and the vector filtered value of the other segment is used as the filtered value. The PM feature can also be used. When a filter is applied to a segment, pixels marked as noise can be ignored. In this paper 4 types of LSVF are considered.

\subsubsection{LSVF1 - LSVMF1 \\ Local Segmentation - TYPE1 \\ Vector Filter - VMF \\ Additional - Not Included}

\subsection{2. $L S V F 2-L S A N F 1$ \\ Local Segmentation - TYPE1 \\ Vector Filter - ANNF \\ Additional - Not Included}

\author{
3.3.3. LSVF1 - LSVMF2 \\ Local Segmentation - TYPE2 \\ Vector Filter - VMF \\ Additional - Pixel Marking in both LS and VF
}

3.3.4. LSVF4 - LSANF2

Local Segmentation - TYPE2

Vector Filter - ANNF

Additional - Pixel Marking in both LS and VF

\section{SIMULATION RESULTS}

In this section, the proposed local segmentation filter structure is evaluated and compared with existing filters. The performance analysis carried out in two ways:

1) A comparison of the classic vector filters (VMF and ANNF) with their proposed variants, LSVMF1, LSVMF2, LSANF1 and LSANF2 in Table 1.

2) A comparison of the proposed LSVF(s) with the stateof-the-art filters. 
Several objective criteria are used to measure the distortion in image reconstruction, which includes the MSE (Mean Square Error), MAE (Mean Absolute Error) and the NCD (Normalized Color Difference). NCD is defined in [3].

\subsection{Impulse Noise Corruption}

The corrupted noise model is assumed to be random impulse noise where the noise term is uniformly distributed over the range of all possible pixel values $(0$ to 255). All images in the simulation are corrupted by channel correlation method proposed by [4]. For example, for a preset percentage of pixels, the R, G or B channel of the pixel value is chosen at random and corrupted by random impulse noise in an independent manner, then a correlation factor $\mathrm{C}=0.5$ is used to introduce more noise into the other color channels for each corrupt pixel. In another word, there is a $50 \%$ chance of further corruption if one channel has been already corrupted.

\subsection{Experimental Performance}

\subsubsection{Experiment One}

In this experiment, the Barbara image was corrupted by $5 \%$ and $10 \%$ of random impulse noise. To evaluate the efficiency of the LS structure, the proposed filters are first compared with their corresponding classic vector filters. Table 1 shows the objective measures for VMF, ANNF and the proposed LSVMF(s) and LSANF(s). It shows that the proposed filters have consistent improvement for both noise levels than their counterparts. Figure 4 demonstrates that the proposed filters perform better at preserving edges and at retaining image structures, with less blurring.

\subsubsection{Experiment Two}

In this experiment, the proposed filters are compared with some of the state-of-the-art filters for the Letter image corrupted by $10 \%$ and $20 \%$ of random impulse noise. In Figure 5, the proposed filters performed well for noise suppression and detail preservation. Most state-of-the-art filters showed unacceptable results as they destroyed lines, edges, and high image detail. Table 2 shows evidence of these degradations.

\section{CONCLUSIONS}

The proposed filters using the LS structure perform better than their classic counterparts. Moreover, it is demonstrated that the PM improves the proposed filters by having higher accuracy in LS and in the reconstruction process. It shows that most filters do not preserve image structure well and even further degrade the image quality even with or without noise. The proposed filters perform better in for image structure preservation than other filters.

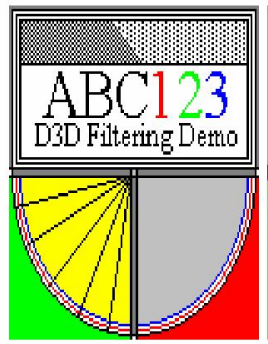

ORIGINAL

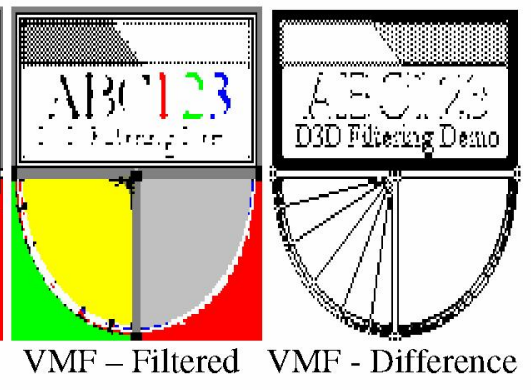

Figure 1: Results of VMF filter for Letter image.

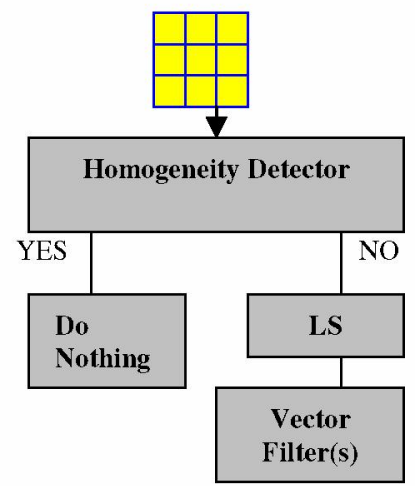

Figure 2: Proposed LSVF structure

\begin{tabular}{|l|c|c|c|c|c|c|}
\hline Noise & \multicolumn{3}{|c|}{ Random Impulse 5\% } & \multicolumn{3}{c|}{ Random Impulse 10\% } \\
Filter & MAE & MSE & NCD & MAE & MSE & NCD \\
\hline None & 7.8 & 922 & 2.87 & 15.6 & 1863 & 5.71 \\
ANNF & 22.6 & $\mathbf{5 9 4}$ & 3.86 & 23.9 & 624 & 4.24 \\
LSANF1 & $\mathbf{1 5 . 6}$ & $\mathbf{3 5 1}$ & $\mathbf{2 . 7 2}$ & $\mathbf{1 8 . 4}$ & $\mathbf{4 7 5}$ & $\mathbf{3 . 4 6}$ \\
LSANF2 & $\mathbf{1 4 . 9}$ & $\mathbf{2 6 8}$ & $\mathbf{2 . 7 0}$ & $\mathbf{1 7 . 0}$ & $\mathbf{3 6 9}$ & $\mathbf{3 . 1 3}$ \\
VMF & 21.2 & $\mathbf{6 2 4}$ & 3.55 & 22.1 & $\mathbf{6 5 6}$ & 3.70 \\
LSVMF1 & $\mathbf{1 4 . 6}$ & $\mathbf{3 6 1}$ & $\mathbf{2 . 5 4}$ & $\mathbf{1 7 . 0}$ & $\mathbf{4 9 0}$ & $\mathbf{3 . 0 7}$ \\
LSVMF2 & $\mathbf{1 3 . 8}$ & $\mathbf{2 8 4}$ & $\mathbf{2 . 5 7}$ & $\mathbf{1 5 . 8}$ & $\mathbf{3 9 0}$ & $\mathbf{2 . 9 7}$ \\
\hline
\end{tabular}

Table 1: Results of color Barbara image. NCD scaled by $10 \mathrm{e}-2$

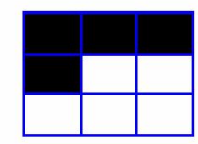

Figure 3: PM - Pixels with known status (black). 


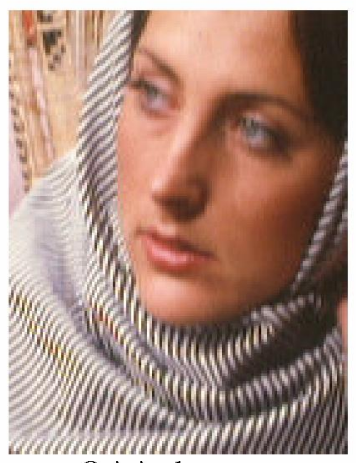

Original

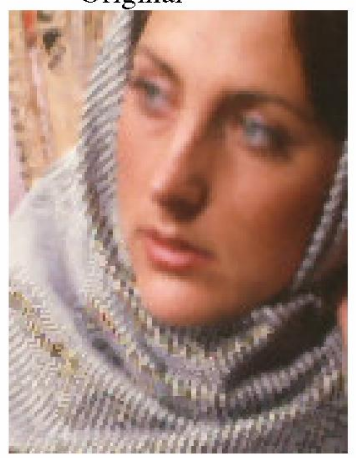

VMF

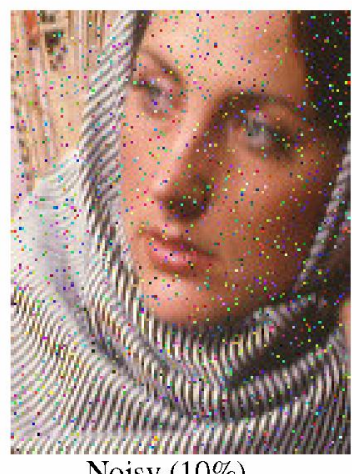

Noisy $(10 \%)$

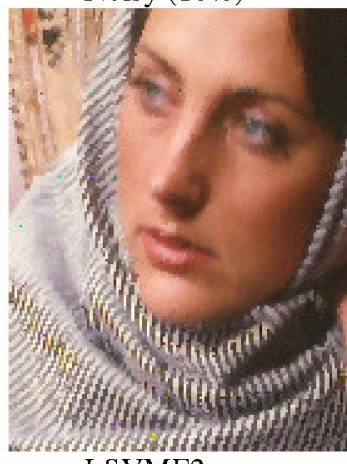

LSVMF2

Figure 4: Results for parts of the Barbara image (10\% noise).

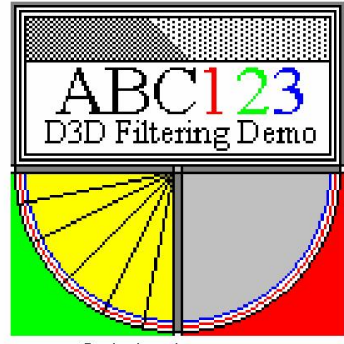

Original

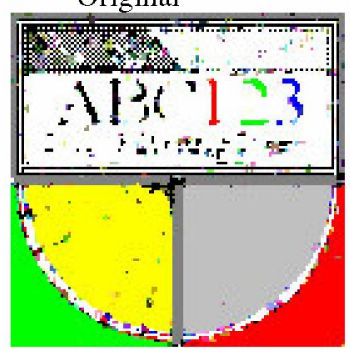

$\mathrm{VMF}$

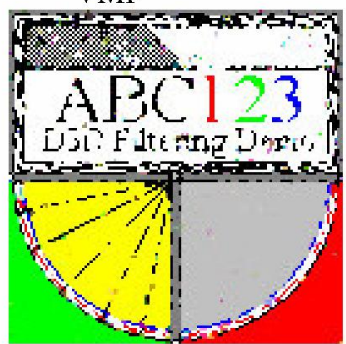

$\mathrm{PGF}$

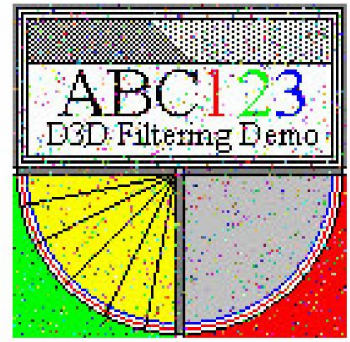

Noisy $(20 \%)$

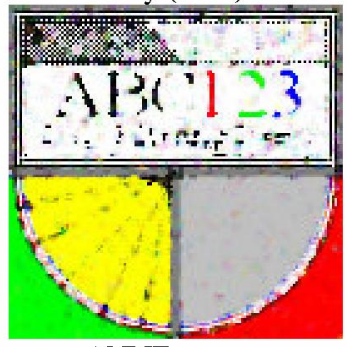

$\mathrm{ANNF}$

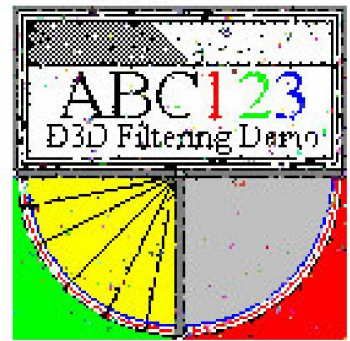

LSVMF2

Figure 5: Results of Letter image (20\% noise)

\begin{tabular}{|c|c|c|c|c|c|c|}
\hline Noise & \multicolumn{3}{|c|}{ Random Impulse 10\% } & \multicolumn{3}{c|}{ Random Impulse 20\% } \\
& MAE & MSE & NCD & MAE & MSE & NCD \\
Filter & & & & & & \\
\hline None & 23.3 & 3794 & 7.3 & 45.5 & 7424 & 14.2 \\
Mean & 182.7 & 22761 & 32.4 & 193.9 & 23208 & 35.4 \\
VMF & 147.5 & 34779 & 27.3 & 150.6 & 34543 & 28.3 \\
GVDF & 142.4 & 32754 & 25.5 & 145.6 & 31954 & 26.3 \\
DDF & 138.5 & 32649 & 25.6 & 143.2 & 32991 & 26.5 \\
HDF & 147.0 & 34089 & 26.4 & 150.2 & 33734 & 26.8 \\
AHDF & 148.8 & 33856 & 26.8 & 151.2 & 32966 & 27.0 \\
FVDF & 145.5 & 32029 & 26.0 & 149.3 & 31211 & 26.9 \\
ANNF & 151.6 & 32587 & 28.3 & 157.7 & 31147 & 29.7 \\
MWC & 132.5 & 24513 & 27.7 & 131.2 & 24614 & 28.0 \\
PGF & 42.1 & 9909 & 7.8 & 70.6 & 16144 & 13.2 \\
LSANF1 & $\mathbf{2 8 . 0}$ & $\mathbf{5 5 1 1}$ & $\mathbf{6 . 5}$ & $\mathbf{4 6 . 4}$ & $\mathbf{8 0 8 8}$ & $\mathbf{1 1 . 4}$ \\
LSANF2 & $\mathbf{2 3 . 7}$ & $\mathbf{5 1 9 6}$ & $\mathbf{5 . 6}$ & $\mathbf{3 7 . 8}$ & $\mathbf{7 4 3 1}$ & $\mathbf{9 . 3}$ \\
LSVMF1 & $\mathbf{2 5 . 6}$ & $\mathbf{5 6 7 6}$ & $\mathbf{5 . 5}$ & $\mathbf{4 1 . 0}$ & $\mathbf{8 3 8 5}$ & $\mathbf{9 . 2}$ \\
LSVMF2 & $\mathbf{2 2 . 5}$ & $\mathbf{5 3 7 3}$ & $\mathbf{4 . 6}$ & $\mathbf{3 4 . 9}$ & $\mathbf{7 8 0 3}$ & $\mathbf{7 . 5}$ \\
\hline
\end{tabular}

Table 2: Results of color Letter image. NCD is scaled by $10 \mathrm{e}-2$

\section{REFERENCES}

[1] R. Lukac, Adaptive Color Image Filtering Based on Center-Weighted Vector Directional Filters, Multidimensional Syst. Signal Process. 15(2), pp.169196,(2004).

[2] I. Pitas and A. N. Venetsanopoulos, Order Statistics in Digital Image Processing, Proceedings of the IEEE, vol. 80 , pp. $1892-1919,(1992)$.

[3] J. Astola, P. Haavisto, Y. Neuvo, Vector median filters. Proc. IEEE, vol.78, pp.678-689,(1990).

[4] P.E. Trahanias, A.N. Venetsanopoulos, Vector directional filters: A New class of multichannel image processing filters. IEEE Trans. Processing, vol.2, No.4, pp.528-534, (1993).

[5] D.G. Karakos, P.E. Trahanias, Combining vector median and vector directional filters: The directionaldistance filters. Proc. ICIP-95, vol.1, pp.171-174,(1995).

[6] M. Gabbouj, F.A. Cheickh, Vector median-vector directional hybrid filter for color image restoration. Proc. EUSIPCO-96, vol. 2, 879-881, Trieste, Italy, Sept. 1996.

[7] K.N. Plataniotis, et.al., An adaptive nearest neighbor multichannel filter. IEEE Trans., on Circuit, Syst., Video Tech., vol.6, no.6, pp.699-703, Dec. (1996).

[8] E. S. Hore, B. Qiu and H. R. Wu, Prediction based image restoration using a multiple window configuration. Optical Engineering, vol.41, pp.1-11, Aug. 2002.

[9] Z. Ma and H.R. Wu, A Neighborhood Adaptive Vector Filter for Color Image Restoration. ISPACS2003, Awaji Island, Japan, December 7-10,(2003).

[10] C. Kenney, Y. Deng, B. S. Manjunath, and G. Hewer, Peer Group Image Enhancement. IEEE Trans. Image Processing, Vol. 6. No. 7, pp. 326-334, Feb. 2001. 